Article

\title{
Look-Ahead Strategies Based on Store-Carry and Forward Relaying for Energy Efficient Cellular Communications
}

\author{
Panayiotis Kolios $^{1, \star}$, Vasilis Friderikos ${ }^{1}$ and Katerina Papadaki ${ }^{2}$ \\ ${ }^{1}$ Centre for Telecommunications Research, King's College London, Strand WC2R 2LS, London, UK; \\ E-Mail: vasilis.friderikos@kcl.ac.uk \\ ${ }^{2}$ Operational Research Group, London School of Economics, Houghton Street WC2A 2AE, London, \\ UK; E-Mail: k.p.papadaki@1se.ac.uk
}

* Author to whom correspondence should be addressed; E-Mail: panayiotis.kolios@kcl.ac.uk; Tel.: +44 (0)20 7848 2889; Fax: +44 (0)20 78482664.

Received: 15 September 2010; in revised form: 25 October 2010 / Accepted: 2 November 2010 / Published: 4 November 2010

\begin{abstract}
With the increasing availability of Internet type services on mobile devices and the attractive flat rate all-you-can-eat billing system, cellular telecommunication networks are experiencing a tremendous growth in data usage demand. However, there are increasing concerns that current network deployment trends (including more efficient radio access techniques and increased spectrum allocation strategies), will be unable to support the increased Internet traffic in a sustainable way. The delay tolerant nature of mobile Internet traffic allows for a large degree of flexibility in optimizing network performance to meet different design objectives and it's a feature that has mostly gone unexplored by the research community. In this paper, we introduce a novel message forwarding mechanism in cellular networks that benefits from the inherent delay tolerance of Internet type services to provide flexible and adjustable forwarding strategies for efficient network operation while guaranteeing timely deliveries. By capitalizing on the elasticity of message delivery deadlines and the actual mobility of nodes inside the cell, considerable performance gains can be achieved by physically propagating information messages within the network.
\end{abstract}

Keywords: energy-efficient communication; store-carry and forward relaying; multihop cellular networks; green networking 


\section{Introduction}

Mobile network operators are currently experiencing an unprecedented increase in data traffic demand. Cisco forecasts that this demand will increase 66 times by year 2013 from the figures in 2008, exceeding the 2 exabytes per month data volume [1]. Already, North American operator AT\&T comments a $5000 \%$ increase in data usage on its network over a three year period, with the growth showing no signs of decreasing in the near future.

To cope with this demand surge, network operators have reside in four main solutions, namely: (1) increasingly smaller cell site deployments, (2) new-higher capacity-radio access technologies (RATs), (3) increased spectrum allocation and (4) network optimization. However, it is anticipated that such measures alone will not be able to keep up with the current data growth rates. For that reason, a significant volume of research work is currently looking into ways of improving the system efficiency so as to drive costs down while maintaining and improving the perceivable user experience. These efforts can be broadly taxonomized into the following two categories:

1. Techniques; including more energy efficient hardware components, physical layer enhancements, energy-aware scheduling, better utilization of available spectrum via cognitive radio techniques and intelligent call admission control algorithms.

2. Architectures; including relay (mostly fixed) aided cellular networks, distributed antenna systems and co-existence of heterogenous mobile and wireless access networks.

The work we present in this paper requires a new architectural framework for cellular networks that will allow a set of mechanisms for store carry and forward relaying to be enabled. In essence, the proposed multihop message forwarding mechanism can be deemed as a generalization of the relay strategies for cellular networks that have been previously considered in the literature. In previous proposals, fixed relay nodes are being deployed within the cell and message forwarding takes place as soon as the relay receives the information (using for example Amplify and Forward (AF) or Decode and Forward (DF) relaying). Instead, here we assume that mobile relay nodes which can postpone the forwarding of received messages in order to reach favorable transmit positions. In this way, the store-carry and forward (SCF) relaying paradigm can be realized within the cell.

Figure 1. A single realization of the store-carry and forward paradigm employed by a mobile relay node within the cell.

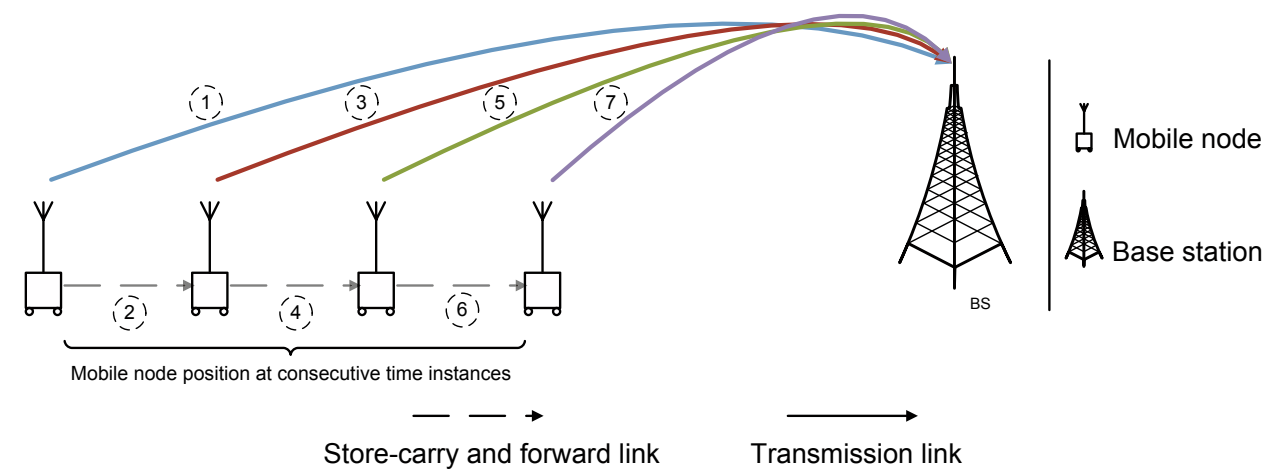


Consider an illustrative example of the above concepts in Figure 1. In this figure a single base stations (BS) entity and a single mobile node at four consecutive snapshots in time are shown. For delay sensitive applications, the direct link path is followed (link 1 in Figure 1). However when the initiated service can tolerate some delay, alternative paths become feasible and can be therefore utilized; either through links $2 \rightarrow 3$ with the mobile node postponing transmission for a single time interval, through links $2 \rightarrow 4 \rightarrow 5$ with delay of two time periods or via route $2 \rightarrow 4 \rightarrow 6 \rightarrow 7$ with delay of three time periods. Clearly, increasing delay tolerance on message delivery deadlines allows for localized transmissions to be realized while the physical propagation of information messages can be achieved through the cell.

The contributions of this paper can be summarized as follows:

1. The store-carry and forwarding paradigm for cellular networks is introduced. While SCF relaying has been studied extensively in the past, the scenario we consider in this work differs dramatically. In all previous occasions, SCF relaying has been considered as the only alternative method for providing communication in intermittently connected networks. In that case because of the absence of an end-to-end path, information was stored for unexpected periods of time before a new contact opportunity arouse. In the case we consider here, end-to-end connectivity is not an issue. On the contrary, due to the presence of the infrastructure nodes there is almost full connectivity within the cell coverage area. However, delay is deliberately expended by mobile nodes while physically propagating information to reach a favorable transmit position. In this way, informed routing decisions are made to satisfy different operating requirements.

2. The benefits of the proposed mode of operation are described and novel network optimization strategies are explained.

3. A theoretical model is detailed for studying the proposed forwarding scheme and a network flow formulation is introduced that is computationally efficient and it can be used in practice to derive optimal forwarding policies.

In the following sections we show how the proposed SCF relaying scheme can be an effective mechanism for message forwarding in cellular networks. Section 2 provides a detailed literature review on the related work. In Section 3 we study a number of potential use cases where SCF relaying can improve system performance, while in Section 4 we formally state the problem and provide a network flow formulation for solving the joint routing and scheduling problem that suffices. Furthermore, Section 5 presents a detailed numerical investigation and in Section 6 considerations are made on the issues that need to be addressed through the protocol stack when delays on the end-to-end paths need to be considered. Finally we conclude the paper in Section 7, where also some future avenues of research are outlined.

\section{Related Work}

Store-carry and forward relaying has been proposed in the past as an effective mechanism to provide connectivity in intermittently connected networks where, either due to the spatial node sparsity in the network or due to the temporal communication patterns, end-to-end connectivity can not be achieved [2-4]. In those cases, a delay in the end-to-end path had to inevitably occur so that the end 
nodes could establish communication. A review of the design objectives and challenges of SCF routing in delay tolerant networks (DTNs) can be found in [5,6].

However for the scenario we consider in this work, delay in the forwarding paths is incurred in a controlled fashion and forwarding paths are formed in a coordinated way. Note that within the cell, the BS provides an almost $100 \%$ coverage. Therefore all nodes can communicate directly with the BS and hence centralized decisions can be taken for the optimal forwarding paths under the different quality of service constraints. In addition, due to the centralized control offered by the BS, messages are not arbitrarily replicated (as it happens most of the cases within sparse networks, using for example epidemic routing [7] to propagate information messages) but the replication is orchestrated by a centralized node, which can be for example the BS. The main problem with epidemic routing is that it consumes a significant portion of network resources in terms of bandwidth and buffering, since information messages need to be copied at every node encounter without any discretion. Therefore, for our envisioned strategies of improving the performance of cellular networks that are discussed here, such a routing scheme is impractical. A number of different works have looked into the problem of more efficient routing in DTNs by reducing the number of copies using several criteria such as the number of copies already send by a node and the mobility pattern of the nodes. To reduce the number of copies the authors in [8] proposed that only the sender could make a copy; this would dramatically reduce the overhead in the network but at the same time message delivery delay could be substantially increased depending on the mobility pattern and actual distance between sender and receiver. An alternative technique that provides copies of messages with a controllable probability has been proposed in [9]. The above schemes assumes isolated mobile nodes and no infrastructure support. In an architectural framework similar to the one we assume in this paper, the authors in [10] explore the potential of infrastructure based wireless networks for aiding store-carry and forward routing. The authors present both analytical and experimental studies and use a variety of routing protocols with emphasis on the RAPID [11] scheme. The Resource Allocation Protocol for Intentional DTN (RAPID) routing protocol estimates the utility of creating a copy of the message (in terms of propagation delay), and copies the message to the node which has the highest value. Further, the authors in [12] devise a routing algorithm that computes shortest paths in DTNs based on the node contact probabilities while satisfying end-to-end reliability constraints. In $[13,14]$ the authors consider the placement of small and inexpensive autonomous wireless nodes (termed as throw-boxes) in between the physical routes of mobile nodes to increase the capacity of the underlay DTN. The authors devised optimal deployment strategies and energy efficient algorithms for information dissemination.

In contrast to the above mentioned cases, for which delay is expended while waiting future contact opportunities, here network connectivity and information communication is not an issue. However, for elastic data services, delay is considered as an additional resource in the system that can be traded off to achieve savings in the communication energy consumption or improvements in the network performance as explained in the sequel.

\section{Possible Use Cases}

Energy efficient operation of cellular networks has become imperative for the sustainability and proliferation of services on mobile devices. However to reduce cost (in terms of the network energy consumption) while maintaining and improving the user experience, considerations need to be taken at 
both the network access side and the radio domain. In a typical energy consumption profile of a European mobile network, more than $50 \%$ of the total energy consumption during operation is attributed to the BS equipment and another $30 \%$ is consumed by the mobile switching and core transmission equipment [15]. Therefore even when the embodied energy to produce the communication equipment is not factored into the cost, shrinking cell sites could not suffice as a viable approach unless efficient operation of the network components is achieved. In [16,17] the authors propose turning off BSs during low utilization periods to save energy while adjacent cells increase their coverage to provide the necessary connectivity. If the BS is operating, then controlling the wireless resources, such as for example the transmit power, has an overall small contribution to the total energy savings. As a result, the most significant energy gains can be achieved by turning off certain BSs when the traffic load in their coverage area is low.

On the other hand, communication over the wireless channel can easily drain the battery of mobile devices. As argued in [18] the energy consumption by the radio frequency (RF) module on a mobile device, is responsible for a large percentage of the total energy consumption budget. Also, transmitting over longer distances consumes considerably more energy as the output transmit power increases more than linearly with the transmission distance. Even worst, it has been shown in [19] that for CDMA (Code Division Multiple Access) cellular networks, the transmit power in the uplink channel increases more than linearly with the network load. Furthermore, in [20] the authors quantified the variability that exists in the communication energy consumption from accessing different RATs, pointing out the high energy efficiency gains of accessing wireless local area (WLAN) networks instead of macrocell sites. However in this case, the availability of WLANs depends heavily on the terminal's location.

In this section we discuss how the proposed SCF relaying scheme could serve as an alternative (and also complimentary) method for reducing the network energy consumption in cellular networks making explicit reference to some of the sources of inefficiency as identified above.

\subsection{SCF Relaying within the Cell}

As briefly introduced in Section 1, via SCF relaying localized transmission are possible when delay on the forwarding information messages can be tolerated. In addition, due to the centralized control offered by the infrastructure units, messages are not replicated at every node encounter and instead forwarding paths are generated with guaranteed deliveries. Within a single cell, the potentials of SCF relaying are considerable. Obviously, allowing mobile relays to carry messages while in transit of their journey reduces the end-to-end communication distance with possible savings in the communication energy consumption. In addition, with reductions in the output transmit power, there is potential to reduce co-channel interference improving even further the system performance. In [21] the authors study the benefits of postponement protocols for the single hop cellular networks. They show that postponing communication could be an effective way for reducing inter-cell interference. Here we extend that previous work for the general multihop scenario where significantly more flexibility on the optimal forwarding policies can be achieved by cooperation between mobile relay nodes.

In the discussion hereafter, we assume that mobile relay nodes are communication units onboard vehicles. Vehicles, which can be private cars or public transport, travel on a road network and thus their mobility patters are defined by the road structure and the density of nodes within the network. Note that inter-vehicle communication has attracted much attention in the past and successful integration with 
cellular networks has been demonstrated [22,23]. With knowledge on location and mobility information, a mobile node can postpone transmission for a later time instance with favorable channel conditions. For the single hop case, a user terminal (UT) onboard a vehicle can refrain communication with the BS such that it moves closer to the cell center. For the general multihop case however, a static UT can transmit to a vehicular relay (VR) which could then carry the message closer to the BS before retransmission. In the same way, a BS can transmit to a VR which could then carry information messages closer to a target user; acting in this way as a message ferry between the two end points. Such an operation is better explained by the topology layout in Figure 2. In this figure we consider the uplink case, however the scenario equally applies to the downlink case as explained above. In the traditional cellular operation, a UT communicates with the BS via the direct link (link 1 in Figure 2). In the basic multihop scheme, a UT could possibly transmit to a relay node (link 2 in Figure 2) which in turn forwards the received data to a relay ahead (link 3 in Figure 2) or to the BS (link 4 in Figure 2). However in the proposed scheme, a relay node can buffer data while in transit (link 5 in Figure 2) allowing for the flexibility to adjust the forwarding strategies depending on the delay sensitivity of message delivery deadlines. Tolerance on message delivery deadlines further allows nodes to buffer information until an effective contact appears for message forwarding. For example, it could be the case that at an instance in time no VRs are present in the vicinity of a target UT. However the BS can inform the UT for future contact opportunities and establish a forwarding path based on both the spatial and temporal dimensions.

Figure 2. Multihop relaying via store-carry and forwarding within a single cell.

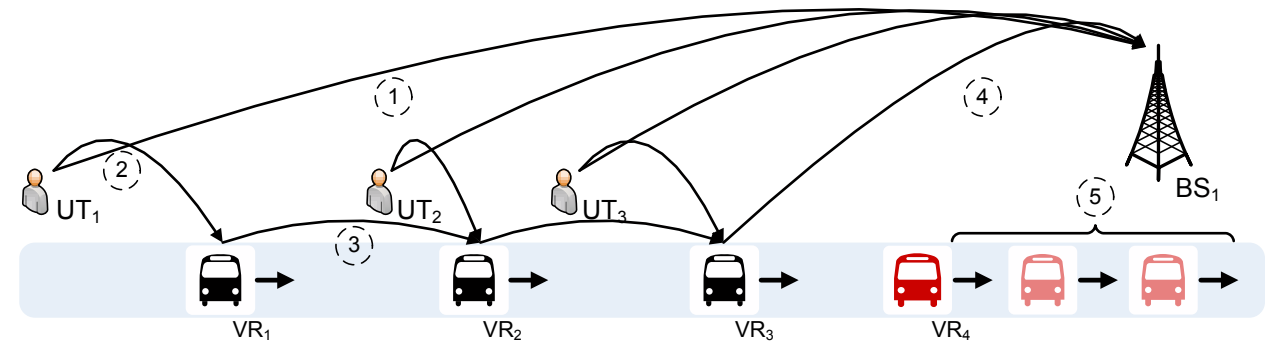

\subsection{SCF Relaying in Multicell Topologies with Homogenous Access Networks}

In the previous section, the single cell operation of SCF relaying was discussed. In this section, by extending the operation of SCF relaying in multicell topologies considerably more opportunities arise. The fundamental difference between previous relaying techniques and the proposed scheme is that non of the past works made use of the inherent mobility of communicating nodes. However, via store-carry and forward relaying, the potential to migrate data traffic from target cells to adjacent cells could be realised by allowing nodes to buffer messages while in transit. In this way, traffic could be distributed across cells to achieve a load balancing effect or shifted to target cells in order for under-utilized cell sites to suspend their operation and save energy. Load balancing in multicell topologies via fixed relaying has been considered in the past [24,25]. However, the performance of the load balancing operation with fixed relaying heavily depends on users' location. For mobile relays on the other hand, spatial dependencies exist between relay nodes and user populations. Consider for example the number of taxis around large crowds. Therefore SCF relaying could be an effective mechanism for load balancing improving both 
the call blocking and call dropping probabilities while increasing at the same time the energy efficiency gains by operating the network at stable loads.

Switching off under-utilized BSs to save energy has been consider in several occasions in the past. The rational behind this work is that only $19-25 \%$ of the BSs carry more than $70-85 \%$ of the total aggregated traffic in the network [26]. Hence, switching off BSs could improve considerably the energy efficiency of the system. In this case, the SCF scheme can provide the necessary connectivity to cell sites where BSs have been switched off without the need for adjacent cells to increase their transmit power in the coverage holes formed by switching off BSs. What's more, instead of UTs transmitting over longer distances when the serving BSs are switching off, the SCF relaying scheme can be employed to shift traffic closer to the cell center, in effect maintaining and/or improving the radio link performance; obviously at the expense of increases message delivery delays.

\subsection{SCF Relaying in Multicell Topologies with Heterogeneous Access Networks}

As mentioned before, with fixed infrastructure node placement, the radio link performance depends greatly on users' location within the serving area. Current trends for future network deployments consider the deployment of wireless local area networks to support the increased capacity requirements and improve the link budget. In [27] the authors propose the use of connectivity maps where UTs are informed of the location of the different access technologies from the serving macrocell BSs. In this way, a UT can switch between the different radio access networks (RANs) depending on its location within the cell. With SCF relaying however, such location dependance has less of an impact in the ability of a UT to send its data through the target RAN. A node can postpone transmission such as a desired location is reached before offloading its data. In Figure 3 one such possible scenario is shown. In the figure the coverage map of two macrosite BSs and three WLAN access points (AP) are shown. In such a multiaccess scenario, traffic can be shifted to the preferred location for the target serving cell to be accessed.

Figure 3. SCF relaying in a multiaccess scenario.

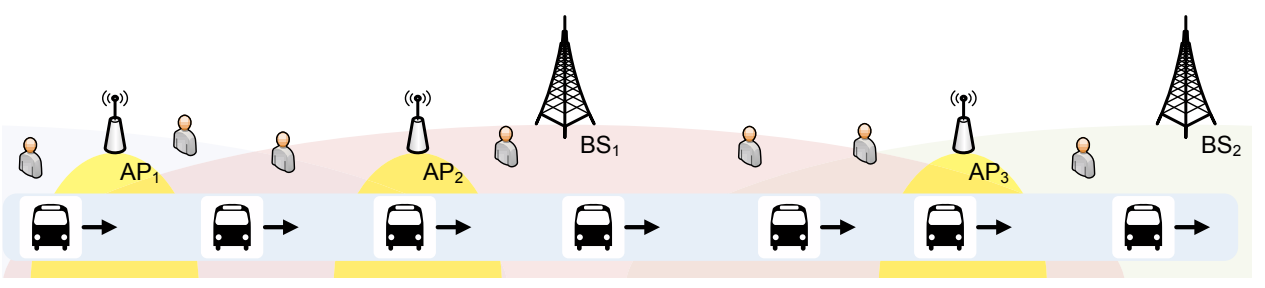

\section{Network Model}

In the previous section some of the potential benefits of SCF relaying were detailed. We argued that when delaying message deliveries does not affect the perceivable user experience, then forwarding paths that employ the store-carry and forward relaying scheme can be realized with great potential for improving system performance in cellular networks. To quantify the potential gains of the proposed scheme we focus in the sequel in the case of a single cell and differ the multicell/multiaccess performance gains as part of a future work. 
Without loss of generality we consider a one dimensional realization of a single cell of radius $R$ meters. Note that for the mathematical formulation of this networking problem that we derive in the sequel, the one dimensional realization assumption is not a necessity; it is merely used for ease of interpretation. For the case of uplink, we assume that there are $\mathcal{M}=\{1, \ldots, M\}$ active users, uniformly distributed across the cell diameter, each with a single message of size $F$ (bits) to communicate to the BS. Further, we consider $\mathcal{N}=\{1, \ldots, N\}$ vehicular relays in each direction of a bidirectional road of length $H=2 R$. For simplicity we assume that all users are static while vehicles travel on the stretch of road with velocity $v_{i}, i \in \mathcal{N}$. Future vehicle positions are estimated using Gipp's car-following model. Note that any other mobility model can be used to capture future vehicle positions. In fact, a highly accurate mobility model will strengthen the robustness of the forwarding decisions being made. Finally we assume that all vehicles have enough buffer capacity to store all messages forwarded to them and that a VR can transmit to, and receive from, a single other VR at an instance in time.

\subsection{Communication Model}

In this work we consider only large scale propagation effects where the pathloss is characterized by an exponential factor to the transmission distance as $D^{\eta}$ where $D$ is the communication range and $\eta$ is the pathloss exponent. Further, we consider that energy is spend by the transmitter and receiver electronics to process the message, $e_{t}$ and $e_{r}$ respectively and energy is required by the power amplifier to transmit the message over the wireless channel, $e_{d}$. Also for all links the transmit data rate is $B$ (bps) and the maximum transmit range is $R$ meters.

Let $\bar{v}_{i j}$ be the relative velocity between two communicating nodes, $i, j$. Then function $g\left(D_{i j}, t\right)$ determines the absolute distance at time $t$ between the transceiver pair $i, j$ during transmission, expressed as follows:

$$
g\left(D_{i j}, t\right)=\left|D_{i j}-\bar{v}_{i j} t\right|
$$

where $D_{i j}$ be the initial distance between the transceiver pair $i, j$ at the start of the transmission. Moreover, the total energy consumption between nodes $i$ and $j$ can be expressed as follows:

$$
f_{i j}=\left(e_{r}+e_{t}\right) B \tau+B e_{d} \int_{0}^{\tau} g\left(D_{i j}, t\right)^{\eta} d t
$$

where $\tau$ is the transmission duration and function $g\left(D_{i j}, t\right)$ accounts for the changing transmission distance as described by (1).

\subsection{Space-time Network}

To construct the possible routing paths in the network, locations of future node positions need to be generated. For that reason we take snapshots in time of length $\tau=\frac{F}{B}$ and replicate node positions at consecutive time instances. Note that both the set of users and the BS are static nodes and thus a single node is required in the network. However for mobile nodes the location in the spatial and temporal dimension are characterized by the terminal ID and the time epoch. Therefore vehicular relay $\mathrm{VR}_{1}$ at the first time instance is described as $\left(\mathrm{VR}_{1}, 1\right)$ while at the second time instance it is defined as $\left(\mathrm{VR}_{1}, 2\right)$. Set 
$V_{p}$ contains all the vehicular nodes at all time periods. In this way a time expanded network is created of the time horizon of interest. Figure 4 a shows the space-time network generated for a single UT and four vehicles. In this figure, vehicles traveling in one direction are shown to aid visualization. It is interesting to note that the space-time network has been considered in the past as the tool to study the performance of routing algorithms in DTNs. In [28] the authors consider the space-time network for sparse networks and suggest the store-carry and forward technique for routing messages. In a similar manner, the work in [29] considers the space-time network with deterministic mobility such that the contact times between nodes can be estimated. Operating on this graph they study the performance of their network instances under different objectives. The clear distinction we try to make here is that for our case, connectivity is not an issue as all nodes can communicate directly with the BS and informed routing decisions can be made. Therefore the space-time network under consideration is not restricted by the contact opportunities as is the case with DTNs. Instead here a densely populated network is considered.

Figure 4. Space-time network for the network model under investigation. (a) The time-expanded network captures node positions at future time instances. Node replication is done every $\tau$ units of time; (b) Connected graph with both transmission and store-carry and forward links.

(a)

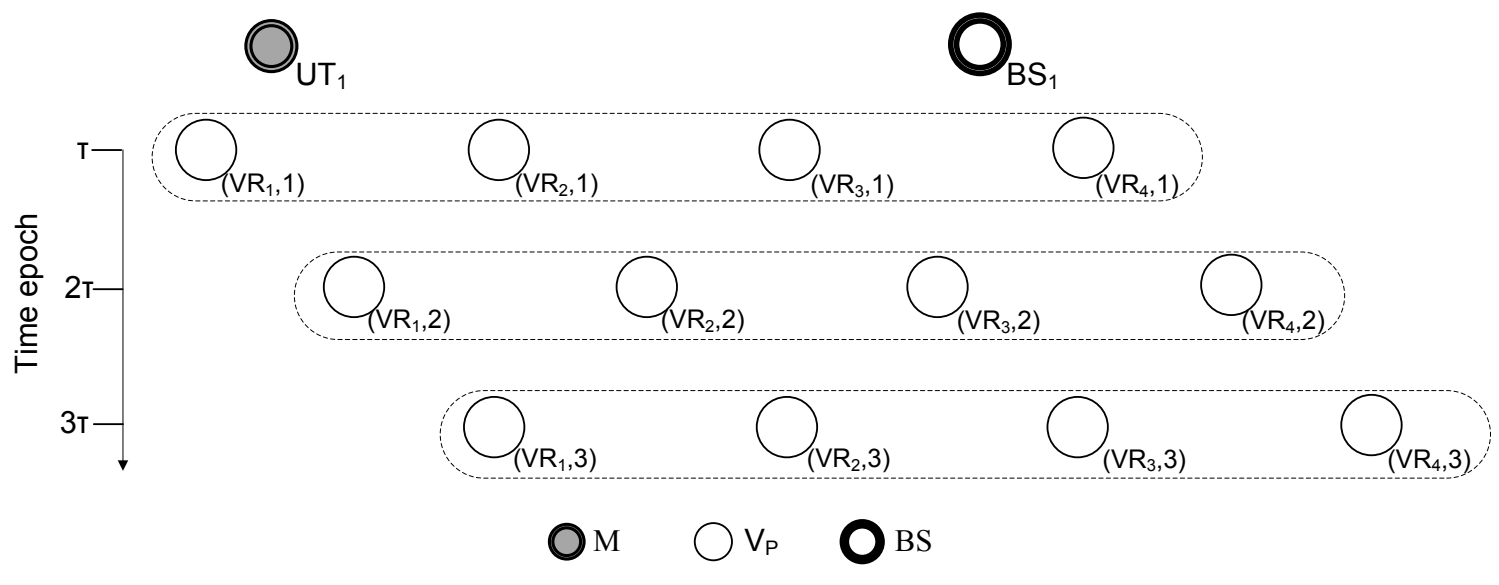

(b)

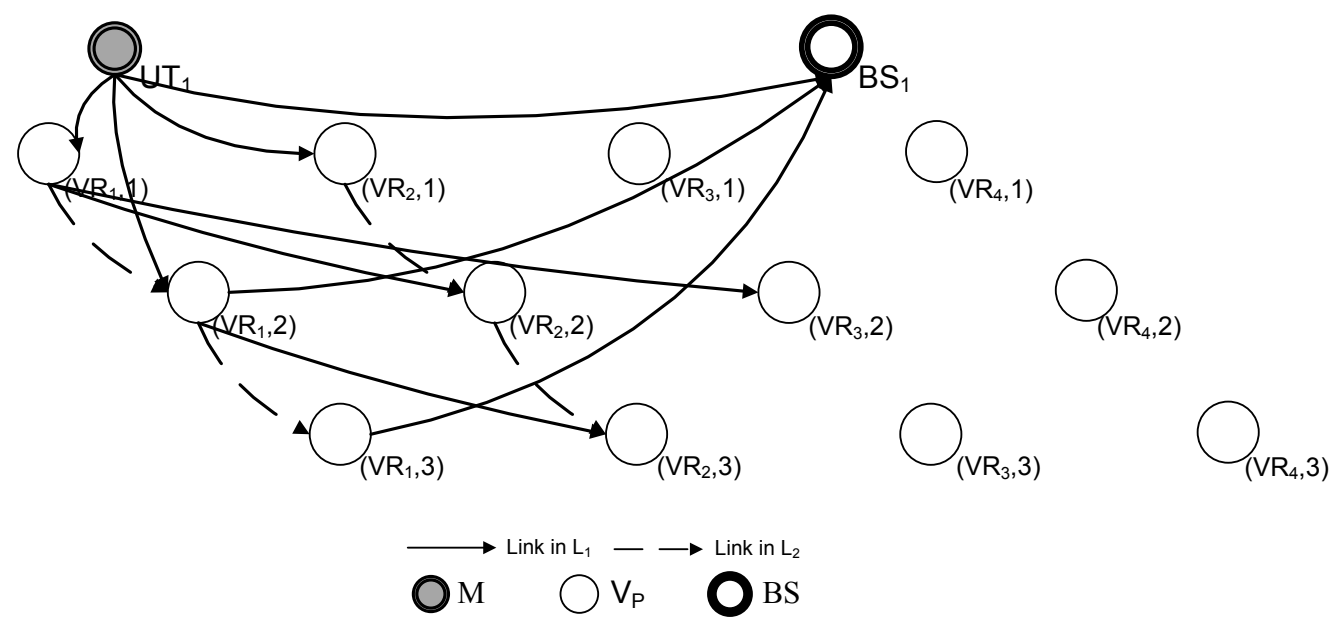


On this network we define the graph $G=(V, L)$ where $V=\mathcal{M} \cup V_{p} \cup B S$ is the set of nodes and $L$ is the set of links containing two subset defined as follows:

$L_{1}$ : set of links that represent transmission of messages between two communicating nodes. Communication can be between vehicles, users and the BS;

$L_{2}$ : set of links where messages are carried from one time period to the next by a mobile node, no communication occurs.

Note that in the space-time network, node replication is done every $\tau$ units of time which is the time needed to transmit a single information message as expressed by Equation (2). As a result, the flow on links in $L_{1}$ can only take binary values. Consider for example the link $\left(\mathrm{VR}_{1}, 1\right) \mapsto\left(\mathrm{VR}_{2}, 2\right)$ in Figure $4 \mathrm{~b}$ with the tail of the link showing the node and time at which the transmission started and the head of the link showing the destination node and transmission termination. By utilizing this link, a single message is forwarded from $\mathrm{VR}_{1}$ to $\mathrm{VR}_{2}$ during the interval $\tau$ to $2 \tau$. On the other hand, link $\mathrm{UT}_{1} \mapsto\left(\mathrm{VR}_{1}, 2\right)$ crosses over 2 time periods. In this case, the users buffers a message for 1 time period and transmits the message during the second time interval. For links in $L_{2}$, a relay node buffers information messages and carries them at consecutive time periods using the actual node mobility (for example, link $\left(\mathrm{VR}_{1}, 1\right) \mapsto\left(\mathrm{VR}_{1}, 2\right)$ in Figure $\left.4 \mathrm{~b}\right)$.

For all links, we define the flow variable $x_{i j} \in \mathbf{Z}$ to be the flow on link $i \mapsto j \in L$. Further, the capacity $u_{i j}$ for all links is defined as follows:

$$
u_{i j}=\left\{\begin{array}{lll}
1 & \mathrm{i} \mapsto \mathrm{j} \in \mathrm{L}_{1} \\
\infty & \mathrm{i} \mapsto \mathrm{j} \in \mathrm{L}_{2}
\end{array}\right.
$$

Note that for links in $L_{1}$ the energy consumption expressed in Equation (2) is a non-linear function with respect to the transmission distance. However for the binary values that the flow variable $x_{i j}$ can attain, the multiplicative cost $x_{i j} f_{i j}$ gives the correct energy consumption values. Furthermore, since one unit of flow should leave each user and reach the BSC, we define the supply and demand for all nodes $i \in V$ as follows:

$$
b(i)= \begin{cases}+1 & \mathrm{i} \in \mathcal{M} \\ -M & \mathrm{i}=\mathrm{BS} \\ 0 & \text { otherwise }\end{cases}
$$

The energy consumed in communication between nodes $i, j \in V$, is as follows:

$$
E(i \mapsto j)= \begin{cases}f_{i j} & \text { for } i \mapsto j \in L_{1} \\ 0 & \text { for } i \mapsto j \in L_{2}\end{cases}
$$

where $f_{i j}$ is defined by (2). The delay per unit flow for all links in $L$ is:

$$
\Phi(i \mapsto j)= \begin{cases}\tau & \text { for } i \mapsto j \in L_{1} \cup L_{2}, \quad i \notin \mathcal{M} \\ (a+1) \tau & \text { for } i \mapsto j \in L_{1}, \quad i \in \mathcal{M}\end{cases}
$$


where for $i \in \mathcal{M}$ and $i \mapsto j \in L_{1}$, the user postpones transmission for $a$ time periods. We define the total communication cost per unit flow as a weighted sum of the energy consumed in transmission and the delay incurred while traversing a link in $L, c_{i j}=E(i \mapsto j)+\lambda \Phi(i \mapsto j)$, where $\lambda$ is a weighting parameter of the delay cost for information to traverse the link $i \mapsto j$.

\subsection{Mathematical Programming Formulation}

To find the optimal routing and scheduling policies for the network flow problem described in the previous section, we formulated the minimum cost integer program described by Equations (7-11) below.

$$
\begin{aligned}
\text { (P1) minimize } & \sum_{i \mapsto j \in L} c_{i j} x_{i j} \\
\text { subject to } & \sum_{j: i \mapsto j \in L_{1}} x_{i j} \leq 1 \forall i, \quad i \in V_{p} \\
& \sum_{k: k \mapsto i \in L_{1}} x_{k i} \leq 1 \forall i, \quad i \in V_{p} \\
& \sum_{j: i \mapsto j \in L} x_{i j}-\sum_{k: k \mapsto i \in L} x_{k i}=b(i) \forall i, i \in V \\
& 0 \leq x_{i j} \leq u_{i j}, x_{i j} \in \mathbf{Z}
\end{aligned}
$$

For problem (P1), the optimal forwarding paths are computed for the competitive parameters of total communication energy consumption and end-to-end message delivery delay. Constraints (8) and (9) are the out-degree and in-degree restrictions for all mobile nodes. Equation (10) is the flow conservation constraint and (11) defines the flow variable. It can be shown that the constraint matrix formed by Equations (8-11) is totally unimodular and thus the linear programming relaxations of this problem is guaranteed to give integer solutions. In effect (P1) can be solved efficiently in time.

\section{Numerical Investigations}

To quantify the possible gains of the proposed SCF relaying scheme we solve problem (P1) described in Section 4.3 to optimality for different values of weighting parameter $\lambda$. For the communication model described in Section 4 we assume that the transceiver fixed circuit power dissipation is $P_{c}=50 \mathrm{~mW}$ (similar to [30]) and the received power threshold for successful communication is $P_{r}=-52 \mathrm{dBm}$ [31]. The data rate for all links is $B=1 \mathrm{Mbps}$ and the antenna height for the transceiver and the receiver is $h_{t}=h_{r}=1.5 \mathrm{~m}$. For these values the following parameters can be deduced: The fixed circuit energy consumption for both the transmitter and receiver is $e_{t}=e_{r}=\frac{P_{c}}{B}$. The energy consumed by the power amplifier is

$$
e_{d}=\frac{P_{r}(4 \pi)^{2}}{B \nu^{2}}
$$

with pathloss exponent $\eta=2$ for ranges less than the threshold distance

$$
d_{\text {brake }}=\frac{4 \pi h_{t} h_{r}}{\nu}
$$


while for larger distances,

$$
e_{d}=\frac{P_{r}}{B h_{t}^{2} h_{r}^{2}}
$$

with pathloss exponent $\eta=4$.

For the network model we assume that $M=\{10,20,30\}$ active users request the uplink of a file of size $F=4$ Mbit to the $\mathrm{BS}$ of radius $R=400 \mathrm{~m}$. Further $N=\{10,20,30,40\}$ vehicles travel along each direction of the stretch of road with maximum speed $v_{\max }=13 \mathrm{~m} / \mathrm{s}$. Figure 5 a shows the energy consumption of the average forwarding path against different message delivery delays. Importantly, for delay insensitive services, the SCF relaying scheme achieves approximately 30 times savings in energy compared to the direct link. Clearly, these energy gains increase with the cell radius as in this case, users have to transmit on average over longer distances on the direct link.

Looking into the performance of the SCF scheme for varying number of candidate forwarding nodes in Figure 5b, it interesting to note that significant savings are achieved even for low vehicle densities. However as the number of active users increases so is the load carried within the buffers of relay nodes which in turn have to start transmitting to the BS from a longer distance; reducing in effect the energy gains. On the other hand, when the number of relays is substantially large, the full potential of SCF relaying can be realized. As seen in Figure $5 b$ the effect on the energy gains of considering increasingly many vehicles within the cell, reduces considerably above a threshold value.

Figure 5. Optimal operating points for the network model under investigation. (a) Energy efficiency gains for increasing number of active users within the cell; (b) Energy efficiency gains for increasing number of candidate forwarding nodes.

(a)

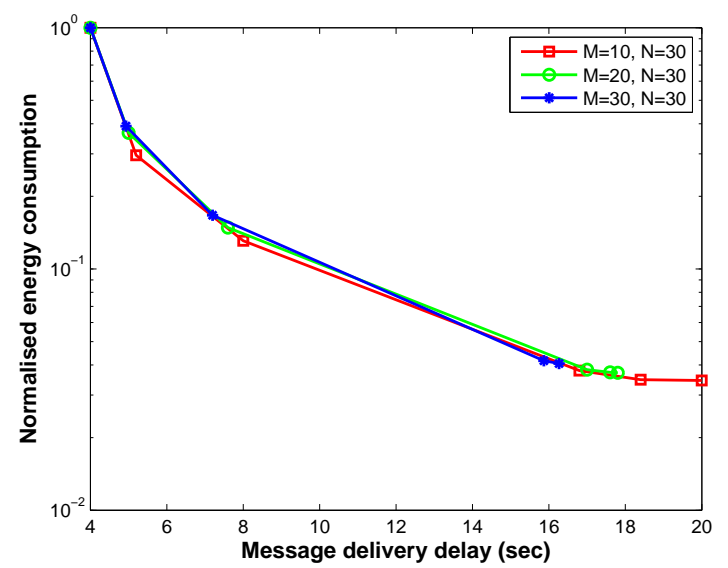

(b)

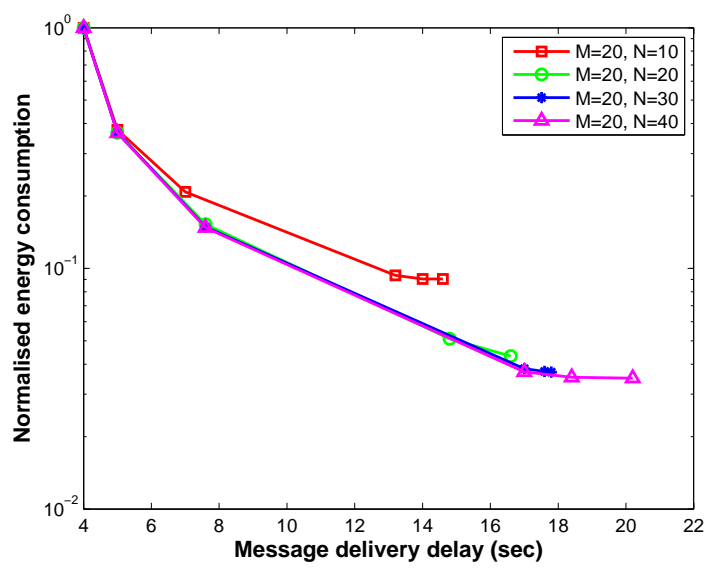

\section{Protocol Stack Considerations}

As shown in the previous section, the performance gains of SCF relaying can be considerable when services can tolerate large delays at the application layer. In order to implement SCF relaying within the cell a number of architectural elements need to be introduced at both the mobile nodes and BSs in the network. A number of different solutions that have been previously proposed and can be utilized to provide such functionalities in cellular networks are outlined in the sequel. In [32] a detailed treatment of the issues arising from such long delays in the end-to-end path have been considered. As described 
by the authors, extended periods of inactivity can cause problems at the transport layer resulting to disconnections. In that work, the authors introduce a DTN architecture that is capable to cope with the unpredictable radio link characteristics. A short term solution has been considered in [33] where the Challenged Internet Access Network Technology Infrastructure (CHIANTI) has been proposed and evaluated. CHIANTI is designed to handle disruptions that could possible occur at different stages of the communication path and that could cause excessive delays. The protocol was evaluated in real network topologies and shown to successfully operate with disruptions of over 5 minutes in length [34]. However, the driving principle in the latter work was to ensure continuation after a disconnection in the end-to-end path. On the contrary, for the work we present here, the delay sensitivity of an information message is determined at the application layer and is a parameter passed and used by the network layer to devise routing paths that satisfy the service deadlines. Therefore, negotiations and guarantees on the delay deadlines should be made by the application layer protocol and the network layer routing protocol.

Already many applications on smartphone devices include the option to adjust the frequency of checking for content updates. In [20] the authors showed that aggregating all update requests and transmitting information in a single shot reduces considerable the communication energy consumption. A plethora also of different applications over the Internet can be deemed as delay tolerant such as for example e-mails, social networking, file transfer and automatic background updates regarding the operating system and/or for different installed software packages. For the proposed relaying scheme, such knowledge on the delay tolerance of application services allows for informed routing decisions to be made at no expense on the perceivable user experience.

\section{Conclusions}

In this paper we introduce a novel message forwarding scheme for cellular networks based on the store-carry and forward paradigm. By trading-off message delivery delays, mobile nodes can postpone message communication for preferable transmit positions. In this way significant performance gains can be realized both in the communication energy consumption and the network operation. The significant energy consumption gains stem from the fact that SCF relaying allows to combat path loss by forwarding the messages when mobile nodes are closer to the BS. Regarding network operation aspects, we showed that the SCF relaying scheme can be an effective mechanism to distribute the load in multicell topologies to achieve either load balancing across the network or reduce the network operating cost by switching off base station units while supporting user connectivity by the alternative store-carry and forward paths.

For the single cell scenario, a mathematical programming formulation was introduced to devise optimal forwarding policies for the different objectives. From the numerical investigations, it was verified that under various network densities, the proposed SCF relaying scheme offered considerable energy efficiency gains. Finally, considerations were made on the requirement for exchange of information between the application and networking layers to facilitate the proposed relaying scheme. Avenues of future research include the characterization of the overall protocol overhead in order to find the message forwarding paths and further investigations using more realistic vehicular mobility models or even utilizing real traces. 


\section{Acknowledgements}

The work reported in this paper has formed part of the Green Radio Core 5 Research Programme of the Virtual Centre of Excellence in Mobile \& Personal Communications, Mobile VCE www.mobilevce.com. This research has been funded by EPSRC and by the Industrial Companies who are Members of Mobile VCE. We are thankful to the anonymous reviewers for their insightful comments and suggestions that helped to greatly improve the presentation of this paper.

\section{References and Notes}

1. Cisco Visual Networking Index: Global Mobile Data Traffic Forecast Update 2009-2014. Cisco White Paper, 2009. Available online: http://www.cisco.com/en/US/solutions/collateral/ ns341/ns525/ns537/ns705/ns827/white_paper_c11-520862.html (accessed on 2 November 2010).

2. Internet Research Task Force (IRTF), Delay-Tolerant Networking Research Group (DTNRG). Available online: http://www.dtnrg.org/ (accessed on 2 November 2010).

3. Fall, K.; Farrell, S. DTN: An Architectural Retrospective. IEEE J. Sel. Area. Commun. 2008, 26, 828-836.

4. Chen, C.; Chen, Z. Exploiting Contact Spatial Dependency for Opportunistic Message Forwarding. IEEE Trans. Mob. Comput. 2009, 8, 1397-1411.

5. Zhang, Z. Routing in intermittently connected mobile ad hoc networks and delay tolerant networks: Overview and challenges. IEEE Commun. Surv. Tutor. 2006, 8, 24-37.

6. Pelusi, L.; Passarella, A.; Conti, M. Opportunistic networking: Data forwarding in disconnected mobile ad hoc networks. IEEE Commun. Mag. 2006, 44, 134-141.

7. Vahdat, A.; Becker, D. Epidemic Routing for Partially-Connected Ad Hoc Networks; Duke Technical Report CS-2000-06; Department of Computer Science, Duke University: Durham, NC, USA. Available online: http://www.cs.duke.edu/ vahdat/ps/epidemic.pdf (accessed on 2 November 2010).

8. Groenevelt, R.; Nain, P.; Koole, G. The message delay in mobile ad hoc networks. Perform. Eval. 2005, 62, 210-228.

9. Matsuda, T.; Takine, T. (p,q)-Epidemic routing for sparsely populated mobile ad hoc networks. IEEE J. Sel. Area. Commun. 2008, 26, 783-793.

10. Banerjee, N.; Corner, M.D.; Towsley, D.; Levine, B.N. Relays, base stations, and meshes: Enhancing mobile networks with infrastructure. In Proceedings of IEEE/ACM Mobicom'08, San Francisco, CA, USA, 14-19 September 2008; pp. 81-91.

11. Balasubramanian, A.; Levine, B.; Venkataramani, A. DTN routing as a resource allocation problem. ACM SIGCOMM Comput. Commun. Rev. 2007, 37, 373-384.

12. Lian, H.E.; Chen C.; Chang J.W.; Shen C.C.; Jan R.H. Shortest path routing with reliability requirement in delay tolerant networks. In Proceedings of International Conference on Future Information Networks, Beijing, China, 14-17 October 2009; pp. 292-297.

13. Zhao, W.; Chen Y.; Ammar M.; Corner M.; Levine B.; Zegura E. Capacity Enhancement using Throwboxes in DTNs. In Proceedings of IEEE International Conference on Mobile Ad hoc and Sensor Systems, Vancouver, BC, Canada, 9-12 October 2006; pp. 31-40. 
14. Banerjee, N.; Corner, M.D.; Levine, B.N. An Energy-Efficient Architecture for DTN Throwboxes. In Proceedings of IEEE International Conference on Computer Communications, Glasgow, UK, 24-28 June 2007; pp. 776-784.

15. Vadgama, S. Trends in Green Wireless Access. Fujitsu Sci. Tech. J. 2009, 45, 404-408.

16. Marsan, M.A.; Chiaraviglio, L.; Ciullo, D.; Meo, M. Optimal Energy Savings in Cellular Access Networks. In Proceedings of IEEE International Conference on Communications Workshops, Dresden, Germany, 14-18 June 2009; pp. 1-5.

17. Zhouy, S.; Gongy, J.; Yangy, Z.; Niuy,Z.; Yang, P. Green Mobile Access Network with Dynamic Base Station Energy Saving. In Proceedings of ACM MobiCom, Beijing, China, 20-25 September 2009.

18. Pentikousis, K. In search of energy-efficient mobile networking. IEEE Commun. Mag. 2010, 1, 95-103.

19. Herhold, P.; Rave, W.; Fettweis, G. Relaying in CDMA networks: Pathloss reduction and transmit power savings. In Proceedings of Vehicular Technology Conference, Jeju Island, Korea, 22-25 April 2003; Volume 3, pp. 2047-2051.

20. Balasubramanian, N.; Balasubramanian, A.; Venkataramani, A. Energy consumption in mobile phones: A measurement study and implications for network applications. In Proceedings of ACM SIGCOMM, Barcelona, Spain, 17-21 August 2009; pp. 280-293.

21. Glisic, S.; Lorenzo, B. Advanced Wireless Networks: Cognitive, Cooperative \& Opportunistic 4G Technology; Wiley Press: Chichester, UK, 2009; pp. 65-69.

22. Mustafa, H.; Zhang, Y. Vehicular Networks: Techniques, Standards, and Applications; CRC Press, Taylor \& Francis Group: Boca Raton, FL, USA, 2009.

23. The ng Connect Program. LTE Connected Car. Available online: http://www.ngconnect.org/ documents (accessed on 2 November 2010).

24. Hongyi, Wu; Chunming, Q.; De, S.; Tonguz, O. Integrated cellular and ad hoc relaying systems: iCAR. IEEE J. Sel. Area. Commun. 2001, 19, 2105-2115.

25. Pabst, R.; Walke, B.H.; Schultz, D.C.; Herhold, P.; Yanikomeroglu, H.; Mukherjee, S.; Viswanathan, H.; Lott, M.; Zirwas, W.; Dohler, M.; Aghvami, H.; Falconer, D.D.; Fettweis, G.P. Relay-based deployment concepts for wireless and mobile broadband radio. IEEE Commun. Mag. 2004, 42, 80-89.

26. Holma, H.; Toskala, A. LTE for UMTS: OFDMA and SC-FDMA Based Radio Access; John Wiley \& Sons, Ltd.: Chichester, UK, 2009; p. 250.

27. Holger, K. An Overview of Energy-Efficiency Techniques for Mobile Communication Systems; Report of AG Mobikom WG7; Technical University Berlin: Berlin, Germany, 2003.

28. Hay, D.; Giaccone, P. Optimal Routing and Scheduling for Deterministic Delay Tolerant Networks. In Proceedings of International conference on Wireless On-demand Network Systems and Services, Snowbird, UT, USA, 2-4 February 2009; pp. 25-32.

29. Merugu, S.; Ammar, M.; Zegura, E. Routing in Space and Time in Networks with Predictable Mobility; Techical Report GIT-CC-04-07; Georgia Institute of Technology: Atlanta, GA, USA, 2004. 
30. Heinzelman, W.B.; Chandrakasan, A.P.; Balakrishnan, H. An application-specific protocol architecture for wireless microsensor networks. IEEE Trans. Wirel. Commun. 2002, 1, 660-670.

31. This is similar to the average signal strength for an LTE receiver at $10 \mathrm{MHz}$ channel bandwidth which is calculated in [26] to be $-56.5 \mathrm{dBm}$.

32. Fall, K. A Delay-tolerant Network Architecture for Challenged Internets; Technical Report IRB-TR-03-003; Intel Research: Berkeley, CA, USA, 2003.

33. Ott, J.; Ylikoski, P.; Seifert, N.; Carroll, C.; Wallbridge, N.; Bergmann, O.; Kutscher, D. The CHIANTI architecture for robust mobile Internet access. In Proceedings of IEEE International Symposium on a World of Wireless, Mobile and Multimedia Networks \& Workshops, Kos, Greece, 15-19 June 2009; pp. 1-9.

34. Bergmann, O.; Gerdes, S. CHIANTI Report on Final User Trials: CHIANTI Deliverable 5.2. Available online: http://www.chianti-ict.org/publications/ (accessed on 2 November 2010).

(C) 2010 by the authors; licensee MDPI, Basel, Switzerland. This article is an open access article distributed under the terms and conditions of the Creative Commons Attribution license (http://creativecommons.org/licenses/by/3.0/.) 